\title{
Screening for Osteoporosis in Older Men: Operating Characteristics of Proposed Strategies for Selecting Men for BMD Testing
}

\author{
Susan J. Diem, MD, MPH', ${ }^{1,2}$ Katherine W. Peters, $M S^{3}$, Margaret L. Gourlay, MD, MPH', \\ John T. Schousboe, MD, PhD ${ }^{5,6}$, Brent C. Taylor, PhD ${ }^{1,2,7}$, Eric S. Orwoll, MD ${ }^{8}$, Jane A. Cauley, DrPH', \\ Lisa Langsetmo, $P h D^{2}$, Carolyn J. Crandall, $M D, M S^{10}$, and Kristine E. Ensrud, MD, MPH ${ }^{1,2,7}$ the \\ Osteoporotic Fractures in Men Research Group
}

\begin{abstract}
'Department of Medicine, University of Minnesota, Minneapolis, MN, USA; ${ }^{2}$ Division of Epidemiology \& Community Health, University of Minnesota, Minneapolis, MN, USA; ${ }^{3}$ California Pacific Medical Center Research Institute, San Francisco, CA, USA; ${ }^{4}$ Department of Family Medicine, University of North Carolina, Chapel Hill, NC, USA; ${ }^{5}$ Park Nicollet Clinic \& HealthPartners Institute, Minneapolis, MN, USA; ${ }^{6}$ Division of Health Policy \&

Management, University of Minnesota, Minneapolis, MN, USA; ${ }^{7}$ Center for Chronic Disease Outcomes Research, VA Health Care System, Minneapolis, MN, USA; ${ }^{8}$ Bone and Mineral Unit, Oregon Health \& Science University, Portland, OR, USA; ${ }^{9}$ Department of Epidemiology, University of Pittsburgh, Pittsburgh, PA, USA; ${ }^{10}$ Department of Medicine, University of California, Los Angeles, CA, USA.
\end{abstract}

BACKGROUND: The optimal approach for selecting men for bone mineral density (BMD) testing to screen for osteoporosis is uncertain.

OBJECTIVE: To compare strategies for selecting older men for screening BMD testing.

DESIGN: Prospective cohort study.

PARTICIPANTS: A total of 4043 community-dwelling men aged $\geq 70$ years at four US sites.

MAIN MEASURES: BMD at the total hip, femoral neck, and lumbar spine using dual-energy $\mathrm{x}$-ray absorptiometry (DXA). Sensitivity, specificity, positive predictive value, negative predictive value, positive likelihood ratio, negative likelihood ratio, and area under the receiver operating curve (AUC) of the Osteoporosis Self-Assessment Tool (OST) and Fracture Risk Assessment Tool (FRAX) without BMD to discriminate between those with and without osteoporosis as defined by World Health Organization (WHO) diagnostic criteria, and between those recommended and not recommended for pharmacologic therapy based on the National Osteoporosis Foundation (NOF) guidelines.

KEY RESULTS: Among the cohort, 216 (5.3\%) had a BMD T-score $\leq-2.5$ at the femoral neck, total hip, or lumbar spine, and 1184 (29.2\%) met criteria for consideration of pharmacologic therapy according to NOF guidelines. The OST had better discrimination (AUC 0.68) than the FRAX (AUC 0.62; $p=0.004$ ) for identifying T-score-defined osteoporosis. Use of an OST threshold of $<2$ resulted in sensitivity of 0.83 and specificity of 0.36 for the identification of osteoporosis, compared to sensitivity of 0.59 and specificity of 0.59 for the use of FRAX with a cutoff of 9.3\% 10year risk of major osteoporotic fracture.

CONCLUSIONS: The OST performs modestly better than the more complex FRAX in selecting older men for BMD testing to screen for osteoporosis; the use of either tool

Electronic supplementary material The online version of this article (doi:10.1007/s11606-017-4153-4) contains supplementary material, which is available to authorized users.

Received September 15, 2016

Revised February 27, 2017

Accepted July 27, 2017

Published online August 16, 2017 substantially reduces the proportion of men referred for BMD testing compared to universal screening. Of 1000 men aged 70 and older in this community-based cohort, the use of an OST cutoff of $<2$ to select men for BMD testing would result in 654 men referred for BMD testing, of whom 44 would be identified as having osteoporosis, and nine with osteoporosis would be missed.

J Gen Intern Med 32(11):1235-41

DOI: $10.1007 / \mathrm{s} 11606-017-4153-4$

(c) Society of General Internal Medicine 2017

\section{INTRODUCTION}

Osteoporosis-related fractures affect one in five men aged 50 years and older globally, ${ }^{1}$ with the incidence of fractures increasing with age; the majority of these fractures occur in men aged 65 years and older. ${ }^{2}$ In 2010 , an estimated $27.9 \%$ of hip fractures occurred in men, and this percentage is expected to rise. ${ }^{1,3}$ Given these data, several organizations ${ }^{4-7}$ have recommended screening for osteoporosis using bone mineral density (BMD) testing in older men. However, there is a lack of data in men on both screening and treatment strategies, resulting in considerable uncertainty about which men to recommend for BMD testing.

Organizations such as the American College of Physicians, National Osteoporosis Foundation (NOF), and Endocrine Society have recommended BMD testing for all men $\geq 70$ years of age. $^{4-7}$ Such a strategy of universal testing naturally identifies all men in this age group with osteoporosis based on World Health Organization (WHO) BMD T-score criteria, ${ }^{8}$ but at considerable cost and based on evidence rated as "low quality" by the Endocrine Society. ${ }^{7}$ There are many knowledge gaps regarding optimal screening strategies. These include 1) a lack of randomized trials demonstrating that screening reduces fractures or fracture-related morbidity or mortality for men or women; 2) limited data in men on pharmacologic therapy for osteoporosis, resulting in greater uncertainty 
regarding appropriate treatment indications and the efficacy of treatment; 3 ) controversies regarding the appropriate reference range for calculation of T-scores for men, with most advocating the use of a female reference range ${ }^{9}$ but others recommending a male reference range, ${ }^{5}$ with resulting discrepancies in classification of men as osteoporotic ${ }^{10}$; and 4) controversy regarding the use of estimated fracture risk to identify candidates for pharmacologic therapy. ${ }^{11}$

In 2011, the US Preventive Services Task Force (USPSTF) concluded that evidence was not sufficient to assess the balance of benefits and harms of osteoporosis screening in men (Grade I). ${ }^{4}$ However, the task force did suggest that men most likely to benefit from BMD testing would have a 10 -year risk for major osteoporotic fracture (MOF) greater than or equal to that of 65-year-old white women, a group for whom screening is widely recommended. This risk is estimated at $9.3 \%$ using the Fracture Risk Assessment Tool (FRAX), a web-based instrument that uses clinical risk factors with and without femoral neck BMD to estimate the 10-year probability of hip and MOF fractures. ${ }^{12}$ Others have suggested a variety of other tools for identifying individuals for screening ${ }^{13}$; the most extensively studied is the Osteoporosis Self-Assessment Tool (OST), with a range of OST score cutoffs recommended to select men for screening. ${ }^{13-18}$ Unlike FRAX, which was developed to predict fracture risk, the OST was developed to predict low BMD and is simple to use. The score is calculated by subtracting the age of the patient in years from the weight in kilograms and multiplying the result by 0.2 .

BMD testing, usually with dual-energy $\mathrm{x}$-ray absorptiometry (DXA), is clinically used to screen for two potential conditions. One is the identification of osteoporosis, defined as a T-score $\leq-2.5$ at the femoral neck, total hip, or lumbar spine; clinical trial data have demonstrated fracture risk reduction with pharmacologic intervention in individuals with T-scores in this range. The other is identification of individuals with Tscores $>-2.5$ but $\leq-1$ (often referred to as osteopenia) and an elevated estimated fracture risk. For individuals with T-scores in this range and with an elevated estimated fracture risk, some guidelines recommend consideration of pharmacologic treatment as well. The NOF and Endocrine Society have suggested that in addition to individuals with osteoporosis, those with osteopenia and a 10 -year risk of a MOF of $\geq 20 \%$ or 10 -year risk of hip fracture $\geq 3 \%$ be considered candidates for pharmacologic intervention. ${ }^{5,7,19}$ The current NOF guidelines recommend the use of a male-specific reference group for the calculation of T-scores in men. ${ }^{5}$

Using data from 4043 community-dwelling men aged $\geq 70$ years enrolled in the Osteoporotic Fractures in Men (MrOS) study, we compared strategies for selecting older men for initial BMD testing to identify men with T-scoredefined osteoporosis and to identify men with osteopenia and an elevated estimated fracture risk. The specified strategies were as follows: screen all men aged 70 and older; screen men with a FRAX (without BMD)-estimated 10-year MOF risk above a range of cutoffs, including 9.3\% (USPSTF strategy); screen men whose OST scores are below a cutoff value ranging from $<-1$ to $<3$.

\section{METHODS}

\section{Study Participants}

From March 2000 through April 2002, 5994 men aged $\geq 65$ years were recruited from population-based listings in six regions of the United States for participation in the baseline examination for MrOS. ${ }^{20,21}$ Men with a history of bilateral hip replacement or who were unable to walk without the assistance of another person were excluded. Men were eligible for this analysis if they were representative of the screened population (i.e., they had no history of non-traumatic hip or clinical vertebral fracture and reported no bisphosphonate or other anti-fracture treatment) and were $\geq 70$ years of age (eFigure 1). The institutional review board at each participating institution approved the study protocol, and written informed consent was obtained from all participants.

\section{Outcomes}

For our primary analysis, we defined the outcome (osteoporosis) as having a BMD T-score of $\leq-2.5$ at the femoral neck, total hip, or lumbar spine, with T-scores calculated using National Health and Nutrition Examination Study (NHANES) III BMD norms for white women aged 20-29, as recommended by the World Health Organization for the diagnosis of osteoporosis in clinical practice. ${ }^{22,23}$

For a secondary analysis, we defined the outcome as a Tscore $\leq-2.5$ at any of the three sites or a T-score $\leq-1.0$ but $>-2.5$ and an elevated estimated fracture risk. We defined elevated estimated fracture risk as a 10 -year risk of a MOF of $\geq 20 \%$ or 10 -year risk of a hip fracture of $\geq 3 \%$ using the FRAX (with BMD) risk calculator. For this outcome, we used a male rather than a female reference group, consistent with the current NOF guidelines. We excluded men with a history of hip or clinical vertebral fracture, as these individuals would already be considered for treatment and would not be considered part of the routine BMD screening population.

\section{Risk Assessment Strategies}

Information regarding osteoporosis risk factors (age, race, rheumatoid arthritis, history of prior fracture, smoking, alcohol intake, and parental history of hip fracture) was obtained from baseline questionnaires. Body weight (balance beam scale at all sites except the Portland site, where a digital scale was used) and height (Harpenden stadiometer ${ }^{24}$ ) were used to calculate BMI in $\mathrm{kg} / \mathrm{m}^{2}$. The FRAX tool was used without a BMD measurement (FRAX [w/o BMD]) to calculate a 10year risk of a MOF (version 3.3). We categorized participants with a FRAX (w/o BMD)-predicted 10-year risk of MOF $\geq 9.3 \%$ as being recommended for BMD testing, as suggested by the USPSTF. 
The OST was calculated as $0.2 \times$ (weight $[\mathrm{kg}]-$ age [yr]), and the score was truncated to yield an integer.

\section{Measurement of BMD}

BMD at the total hip, femoral neck, and lumbar spine was measured at baseline using dual-energy x-ray absorptiometry (DXA) with Hologic QDR 4500 W scanners (Hologic, Inc., Bedford, MA). A central quality-control laboratory, certification of DXA technicians, and standardized procedures for scanning were implemented to ensure reproducibility of DXA measurements. ${ }^{21}$

\section{Other Measurements}

Participants attending the clinic examinations were asked to bring all current (any use within the last 2 weeks) prescription and nonprescription medications with them. Interviewers completed a medication history for each participant, including name of medication and frequency of use. Current use of bisphosphonates, other anti-fracture treatments, and oral corticosteroids was determined using a computerized dictionary to categorize type of medication from product brand and generic names obtained from containers. ${ }^{25}$

\section{Statistical Analysis}

We calculated the proportion of men who would be selected for BMD testing using a range of cutoffs for each prescreening strategy (OST and FRAX [w/o BMD]). We also calculated the proportion of men with a BMD T-score $\leq-2.5$ and the proportion of men who would be recommended for pharmacologic therapy based on NOF guidelines that would be identified by each of these strategies.

We calculated the sensitivity, specificity, positive predictive value (PPV), negative predictive value (NPV), positive likelihood ratio (LR+), negative likelihood ratio (LR-), the number of missed cases of T-score-defined osteoporosis, (false negatives), and the number of DXAs performed on men without osteoporosis (false positives) for the identification of men with osteoporosis for a range of cutoffs for each of the strategies. We used a female reference group for calculation of T-scores for this outcome. We performed similar analyses for the identification of men with osteoporosis or osteopenia and an elevated estimated fracture risk, using a male reference group. ${ }^{26}$ In addition, we plotted the receiver operating characteristic (ROC) curves for the detection of these outcomes across a range of possible thresholds for the OST and the FRAX (w/o BMD) and calculated the area under the curve (AUC). ${ }^{27}$

\section{RESULTS}

\section{Baseline Characteristics}

Baseline characteristics of the study population are presented in Table 1. Using a female and male reference group for T- score $\leq-2.5$ (at the femoral neck, total hip, or lumbar spine), $216(5.3 \%)$ and 397 (9.8\%) men, respectively, were classified as having osteoporosis. A total of $787(19.5 \%)$ men had a Tscore $\leq-1.0$ but $>-2.5$ at one or more of the three sites and an estimated fracture risk at or above the NOF FRAX (with BMD) intervention thresholds. Based on NOF guidelines, $1184(29.3 \%)$ men in the study population would be classified as eligible for drug treatment (Table 1).

\section{Comparison of Risk Assessment Strategies}

The proportion of the study population who would be referred for BMD testing ranged from $23.5 \%$ for an OST cutoff of $<-1$ to $100 \%$ for the strategy of screening all men 70 years and older. The FRAX (w/o BMD) tool (10-year risk of MOF estimated at $\geq 9.3 \%$ ) identified $42.2 \%$ of men for BMD testing. Of the 216 men with T-score-defined osteoporosis using a female reference group, $76.9 \%$ were identified using an OST cutoff of $<1,82.4 \%$ were identified using an OST cutoff of 2 , and $59.3 \%$ were identified using a FRAX (w/o BMD) with a cutoff of $\geq 9.3 \%$ (Table 2). Results were similar when a male reference group was used for the calculation of T-scores.

The receiver operating characteristic (ROC) curves for the OST- and FRAX (w/o BMD)-estimated 10-year risk of a MOF over their full ranges for the prediction of osteoporosis are shown in Fig. 1a. The OST had a higher AUC (0.68 vs. 0.62$)$ than the FRAX (w/o BMD) $(p=0.004)$.

Table 3 shows the sensitivity, specificity, PPV, NPV, LR+, LR-, and the number of false negatives (missed cases) and false positives (unnecessary DXA tests) for a range of cutoffs of the OST- and FRAX (w/o BMD)-estimated risk for predicting osteoporosis based on a female reference group. The strategy of testing men with a FRAX (w/o BMD)-estimated 10 -year risk of a MOF of $\geq 9.3 \%$ had sensitivity of 0.59

Table 1 Baseline Characteristics of Men Aged 70 Years and Older, Non-Traumatic Hip or Spine Fracture Excluded

\begin{tabular}{|c|c|}
\hline Characteristic & $N=4043$ \\
\hline Age at enrollment, years, mean (SD) & $76.3(4.8)$ \\
\hline White (non-Hispanic), no. (\%) & $3669(90.8)$ \\
\hline Body mass index (BMI), $\mathrm{kg} / \mathrm{m}^{2}$, mean $(\mathrm{SD})$ & $27.1(3.7)$ \\
\hline Underweight/normal weight (BMI <25), no. (\%) & $1180(29.2)$ \\
\hline Overweight (BMI 25-30), no. (\%) & $2084(51.6)$ \\
\hline Obese $(\mathrm{BMI} \geq 30)$, no. $(\%)$ & $779(19.3)$ \\
\hline$>10 \%$ weight loss since age 25, no. $(\%)$ & $158(3.9)$ \\
\hline Antiandrogen use, no. (\%) & 159 (3.9) \\
\hline \multicolumn{2}{|l|}{ Smoking status, no. $(\%)$} \\
\hline Current & $99(2.4)$ \\
\hline Past & $2419(59.6)$ \\
\hline Never & $1536(38.0)$ \\
\hline Alcohol consumption $\geq 21$ drinks per week, no. (\%) & $151(3.7)$ \\
\hline History of parental fracture, no. (\%) & $484(12.0)$ \\
\hline Rheumatoid arthritis, no. (\%) & $219(5.4)$ \\
\hline Oral corticosteroid use, no. (\%) & $75(1.9)$ \\
\hline Osteoporosis*, no. (\%) using female reference group & $216(5.3)$ \\
\hline Treatment recommended per NOF guidelines ${ }^{\dagger}$, no. (\%) & $1184(29.3)$ \\
\hline \multicolumn{2}{|c|}{$\begin{array}{l}\text { NOF National Osteoporosis Foundation } \\
* \text {-score } \leq-2.5 \text { at femoral neck, total hip, or lumbar spine } \\
+T \text {-score } \leq-2.5 \text { at any site using a male-specific reference group, or } \\
\text { major osteoporotic fracture (MOF) } \geq 20 \% \text { or hip fracture risk } \geq 3 \% \text { and } \\
\text { osteopenia (NOF) }\end{array}$} \\
\hline
\end{tabular}


Table 2 Proportion of Men Recommended for Screening by Proposed Strategies and T-Score* Category

\begin{tabular}{lll}
\hline \hline Strategy & $\begin{array}{l}\text { Overall, no. }(\%) \\
(\boldsymbol{n}=\mathbf{4 0 4 3})\end{array}$ & $\begin{array}{l}\text { T-score* } \leq-\mathbf{2 . 5} \text { at } \\
\text { least one anatomic } \\
\text { site, no. (\%) } \\
(\boldsymbol{n}=\mathbf{2 1 6})\end{array}$ \\
\hline Men $\geq 70$ years & $4043(100)$ & $216(100)$ \\
OST <-1 & $953(23.5)$ & $102(47.22)$ \\
OST $<0$ & $1485(36.6)$ & $137(63.43)$ \\
OST $<1$ & $2040(50.3)$ & $166(76.85)$ \\
OST $<2$ & $2619(64.6)$ & $178(82.41)$ \\
OST $<3$ & $3076(75.8)$ & $192(88.89)$ \\
FRAX (w/o BMD) ${ }^{\dagger} \geq 7 \%$ & $2720(67.28)$ & $174(80.56)$ \\
FRAX (w/o BMD) $\geq 8 \%$ & $2215(54.79)$ & $154(71.3)$ \\
FRAX (w/o BMD) $\geq 9 \%$ & $1820(45.02)$ & $134(62.04)$ \\
FRAX (w/o BMD) $\geq 9.3 \%$ & $1718(42.4)$ & $128(59.26)$ \\
FRAX (w/o BMD) $\geq 10 \%$ & $1465(36.24)$ & $114(52.78)$ \\
\hline
\end{tabular}

OST Osteoporosis Self-Assessment Tool, FRAX Fracture Risk Assessment Tool, BMD bone mineral density

*T-scores calculated using female reference group

tFRAX tool used without a BMD measurement to calculate a 10-year risk of a major osteoporotic fracture (MOF)

and specificity of 0.59 ; an OST score of $<1$ had sensitivity of 0.77 and specificity of 0.51 , while an OST score $<2$ had sensitivity of 0.83 and specificity of 0.36 .

The use of the OST cutoff of $<2$ to identify men for testing would result in missing 9 of 53 cases of osteoporosis per 1000 men, while reducing the number of men referred for BMD testing who would not have osteoporosis to $601 / 1000$ (Table 3).

For the detection of men with osteoporosis or osteopenia with elevated fracture risk (using a male reference group), the ROC curves for the OST and FRAX (w/o BMD) for the prediction of this outcome are shown in Figure 1b. The FRAX (w/o BMD) had a higher AUC (0.79 vs. $0.68 ; p<0.001)$ than the OST for this outcome. Sensitivity, specificity, PPV, NPV, $\mathrm{LR}+, \mathrm{LR}-$, and the number of false negatives (missed cases) and false positives (unnecessary DXA tests) for a range of cutoffs of the OST- and FRAX (w/o BMD)-estimated risk are shown in Table 4.

\section{DISCUSSION}

We found a low prevalence of T-score-based osteoporosis among men, consistent with data from NHANES 2005$2010 .{ }^{28}$ Using a female reference group to calculate T-scores, $5.3 \%$ of these community-dwelling men $\geq 70$ years of age had a T-score $\leq-2.5$ at one or more of the three anatomic sites. An additional $4.5 \%$ of the cohort would be classified as having osteoporosis if a male-specific reference group were used to calculate the T-scores. Because men on average have higher BMD than women, the use of a male reference group to calculate T-scores results in men being classified as having a $\mathrm{T}$ score $<-2.5$ at a higher absolute BMD and thus at a lower risk of fracture. In 2013, the International Society for Clinical Densitometry formally recommended the use of a female reference group for the calculation of T-scores for men and women. ${ }^{29}$

Our data make explicit the trade-offs clinicians face when deciding whether to implement a universal testing strategy for men $\geq 70$ years of age or to utilize a pre-screening tool to identify men for BMD testing. Use of the OST with a cutoff of 1 or 2 identified $77 \%$ and $83 \%$ of men with osteoporosis, respectively, while use of the FRAX (w/o BMD)-predicted 10-
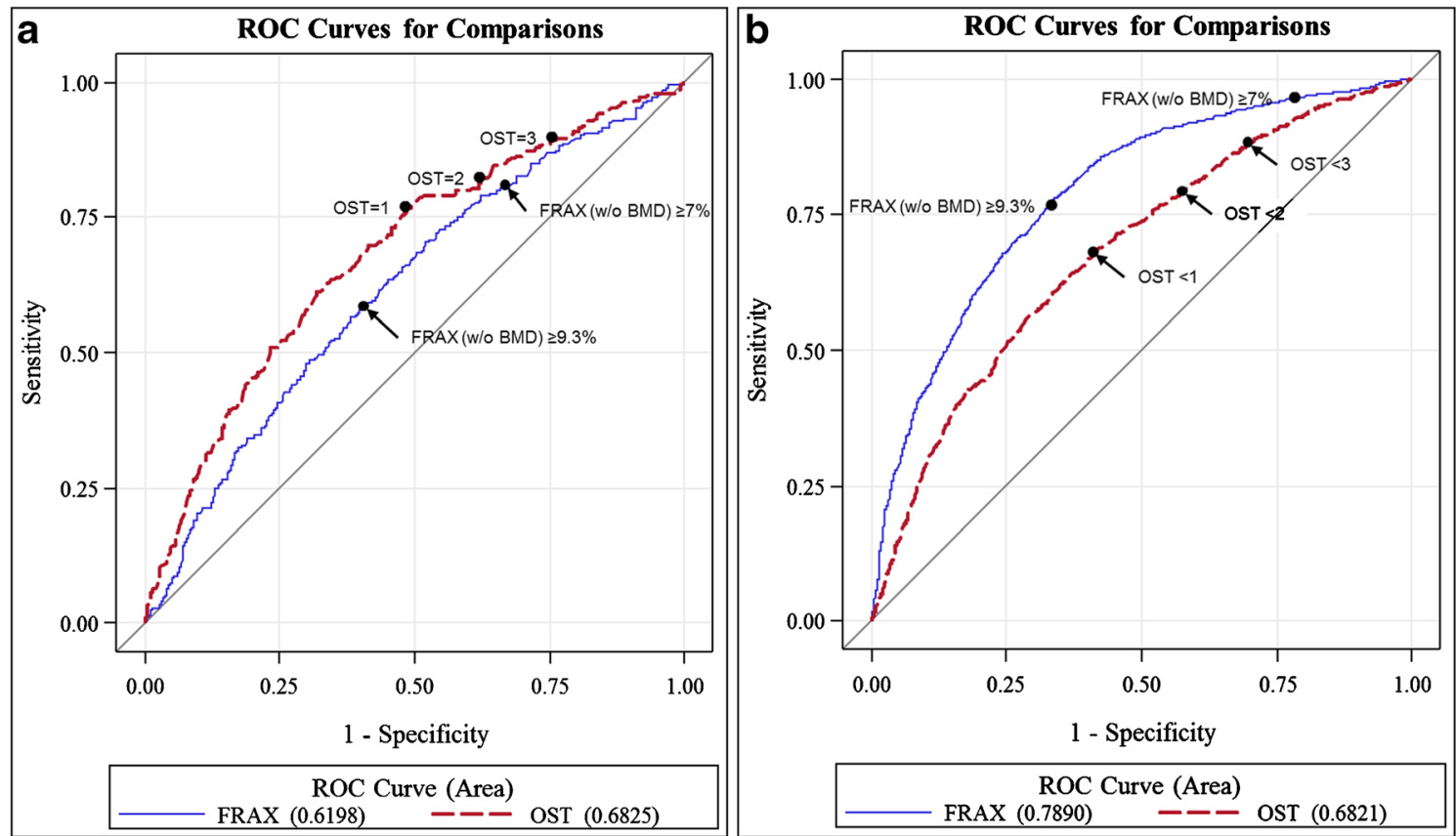

Figure 1 a ROC curves for the OST and FRAX (w/o BMD) over a range of values for the prediction of FN, TH, or LS T-score $\leq-2.5$. b ROC curves for the OST and FRAX (w/o BMD) over a range of values for the prediction of men identified for treatment based on NOF guidelines. The black diagonal line indicates a reference $\mathrm{AUC}$ of 0.50 (no better than chance alone). $R O C$ receiver operating characteristic, OST Osteoporosis Self-Assessment Tool, FRAX Fracture Risk Assessment Tool, FN femoral neck, TH total hip, $L S$ lumbar spine, NOF National Osteoporosis Foundation, MOF major osteoporotic fracture, $A U C$ area under the curve. 

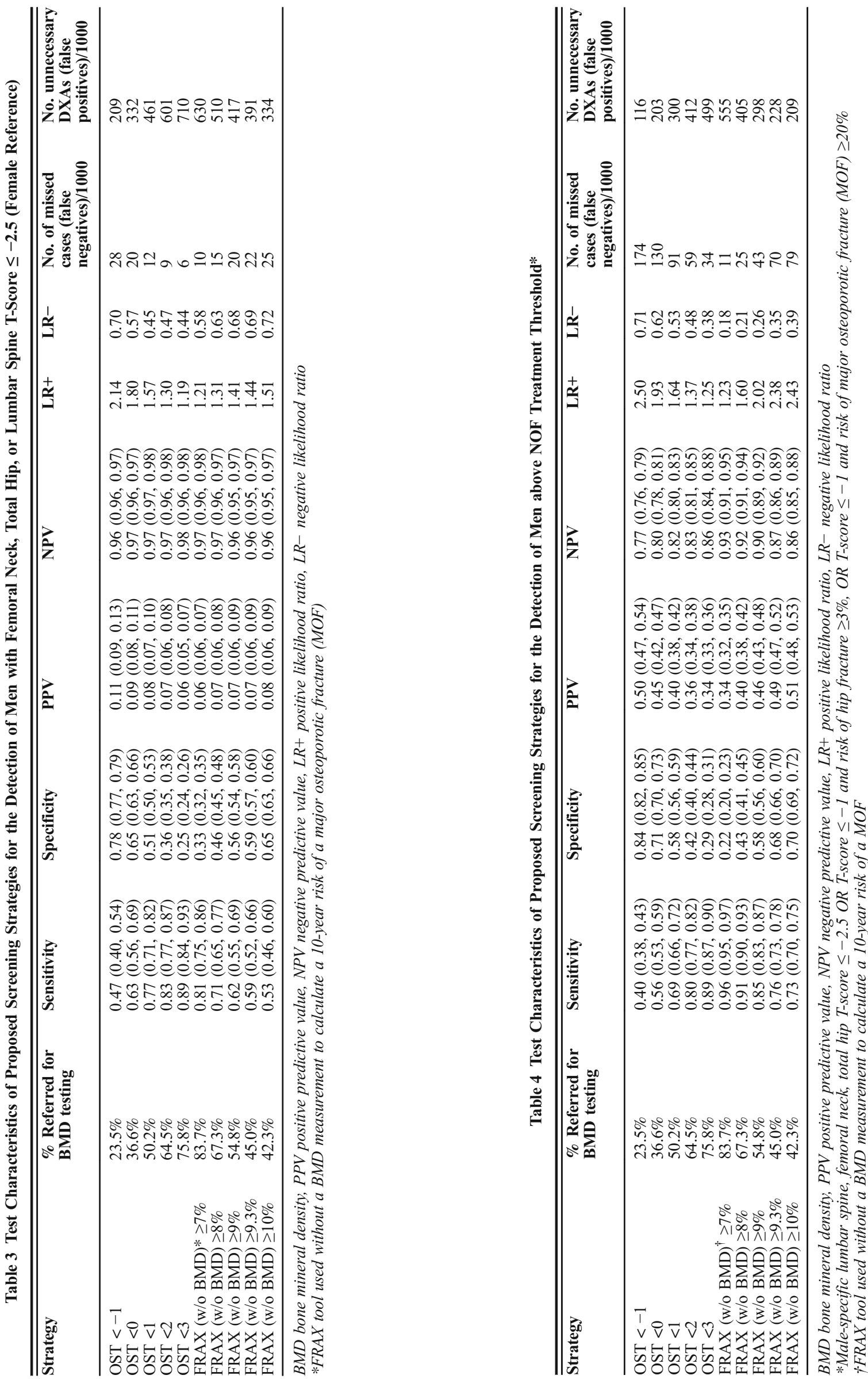
year risk of $\mathrm{MOF} \geq 9.3 \%$, as suggested by the USPSTF, identified 59\% of men with BMD-defined osteoporosis.

The positive LRs for all cutoffs for the OST and FRAX (w/o BMD) studied for the prediction of T-score-defined osteoporosis are relatively small; however, the negative LRs for an OST cutoff of 1-3 are in a range (0.4-0.5) that may be clinically useful for identifying men who should not be referred for BMD testing. ${ }^{30}$ Although negative LRs in this range for a diagnostic test are relatively weak, these tools are not being used as a diagnostic test, but rather to screen men to determine whom to refer for testing with DXA; in this setting, negative LRs in this range may be reasonable.

The pre-screening strategy based on FRAX (w/o BMD) performed better than pre-screening based on OST for the detection of those who would be considered for pharmacologic therapy if the indications for treatment were extended to those with osteopenia and an elevated estimated fracture risk, as suggested by NOF guidelines. This result is to be expected given that the NOF guidelines include an elevated FRAX score with BMD as an indication for treatment. This highlights the unique position of FRAX: FRAX was developed as a treatment decision tool to identify individuals at higher risk of fracture; its use as a pre-screening tool to select individuals for BMD testing was not its original purpose, and may lead to more men being considered for treatment. In this cohort, nearly $30 \%$ of men aged 70 years and older would meet NOF criteria for pharmacologic treatment.

Our results are similar to those of Pang et al., who found that the simpler OST performed better than FRAX (w/o BMD) for identifying osteoporosis in a mixed-gender cohort of 626 adults aged 70 years and older. ${ }^{31}$ The OST, although most extensively evaluated in women, has been evaluated in several studies in men. ${ }^{13-16,18,32}$ In a recent systematic review and meta-analysis of the performance of several clinical risk assessment instruments for osteoporosis screening, the OST performed as well as or better than more complex instruments in identifying individuals with BMD-defined osteoporosis. ${ }^{13}$

A previous study of the MrOS cohort that included all men aged $\geq 65$ years used a male-specific reference for the calculation of BMD T-scores ${ }^{15}$ and reported sensitivity of 0.88 for an OST cutoff of $<2$ in identifying osteoporosis. In addition to the difference in reference groups, our work also differs from the earlier work in that we have extended it to examine the use of these pre-screening strategies in selecting men for DXA testing when the outcome of interest incorporates fracture risk estimates, as recommended by several organizations.

Cost-effectiveness analyses have not supported a universal BMD testing approach for all men $\geq 70$ years of age, ${ }^{33}$ although one found that body weight could be used to identify older men without prior fracture for whom BMD testing would be cost-effective. ${ }^{34}$ Another analysis ${ }^{35}$ found that using the OST to select high-risk men for testing improved the costeffectiveness of performing DXA in older men. None of the existing cost-effectiveness analyses examined the effect of expanding treatment indications to include men with osteopenia and an elevated estimated fracture risk.

The study has several strengths, including the large, wellcharacterized cohort and the measurement of BMD in all participants. However, the population studied was predominantly white, thus limiting generalizability; in addition, participants were recruited from the community and may be healthier than other populations.

Those guidelines that recommend pharmacologic treatment for individuals with osteopenia (T-score $\leq-1.0$ but $>-2.5$ ) and elevated fracture risk do so based on findings from one costeffectiveness study ${ }^{36}$; no randomized clinical trial utilizing these treatment thresholds has been conducted to date. Treatment recommendations that call for consideration of pharmacologic intervention in those with a T-score $\leq-2.5$ at the femoral neck, total hip, or lumbar spine, on the other hand, are based on rigorous clinical trial data, albeit primarily in postmenopausal women.

Given the low prevalence of osteoporosis in communitydwelling older men, as well as the uncertainty surrounding the use of estimated fracture risk to guide treatment decisions, a universal screening approach results in BMD testing in many men who do not have osteoporosis and the potential overidentification of candidates for pharmacologic therapy for whom therapy is of uncertain benefit. While most older men who sustain a fracture do not have pre-existing osteoporosis, ${ }^{37}$ the evidence that existing pharmacologic treatments, all of which address skeletal health, reduce fracture risk in men without osteoporosis is limited.

Among the strategies examined, the use of an OST cutoff of $<2$ substantially reduced the proportion of men screened, reduced the number of false positives while missing few cases of T-score-defined osteoporosis, and performed better than the more complex FRAX (w/o BMD) strategy. Further research toward developing targeted screening of men and additional trials of pharmacologic therapy in men to better characterize appropriate candidates for treatment are critical for developing targeted screening strategies for men or for creating the evidence base needed to support universal BMD testing in men.

Corresponding Author: Susan J. Diem, MD, MPH; Division of Epidemiology \& Community HealthUniversity of Minnesota, Minneapolis, MN, USA (e-mail: sdiem@umn.edu).

Author Contributions: Susan J. Diem - interpretation of data and preparation of manuscript.

Katherine W. Peters - analysis and interpretation of data, critical review of manuscript.

Margaret L. Gourlay - interpretation of data, critical review of manuscript.

John T. Schousboe - interpretation of data, critical review of manuscript.

Brent C. Taylor - interpretation of data, critical review of manuscript. Eric S. Orwoll - study concept and design, acquisition of subjects and data, interpretation of data, critical review of manuscript.

Jane A. Cauley - study concept and design, acquisition of subjects and data, interpretation of data, critical review of manuscript. 
Lisa Langsetmo - interpretation of data, critical review of the manuscript.

Carolyn J. Crandall - interpretation of data, critical review of manuscript.

Kristine E. Ensrud - study concept and design, acquisition of subjects and data, interpretation of data, critical review of manuscript.

Funding Source: The Osteoporotic Fractures in Men (MrOS) Study is supported by funding from the National Institutes of Health. Support is provided by the following: National Institute on Aging (NIA), National Institute of Arthritis and Musculoskeletal and Skin Diseases (NIAMS), National Center for Advancing Translational Sciences (NCATS), and NIH Roadmap for Medical Research (under grant numbers UO1 AG027810, UO1 AG042124, UO1 AG042139, UO1 AG042140, UO1 AG042143, UO1 AGO42145, UO1 AG042168, UO1 AR066160, and UL1 TROO0128). Dr. Gourlay is supported by the NIA, grant number R01 AG046294. The funding agencies had no direct role in the conduct of the study; the collection, management, analysis, or interpretation of the data; or preparation or approval of the manuscript.

This manuscript is the result of work supported with resources and use of facilities of the Minneapolis VA Health Care System. The contents do not represent the views of the U.S. Department of Veterans Affairs or the United States government.

\section{Compliance with Ethical Standards:}

Conflict of Interest: Dr. Orwoll has served as consultant to Merck, Eli Lilly, and Amgen and has received grants from Merck and Eli Lilly in the last three years. Dr. Cauley has served as consultant on a Merck advisory board and has received honoraria from Merck in the last three years. Dr. Ensrud has served as a consultant on the Merck, Sharp \& Dohme advisory board. All other authors declare that they do not have a conflict of interest.

Prior Presentations: A portion of these findings were presented in abstract form at the annual meeting of the Society of General Internal Medicine in May 2015.

\section{REFERENCES}

1. Ebelin P. Osteoporosis in men: why change needs to happen. Mitchell P, editor. www.iofbonehealth.org . Updated 2014. International Osteoporosis Foundation. Accessed June 9, 2017

2. Burge R, Dawson-Hughes B, Solomon DH, Wong JB, King A, Tosteson A. Incidence and economic burden of osteoporosis-related fractures in the United States, 2005-2025. J Bone Miner Res. 2007;22:465-75.

3. Stevens JA, Rudd RA. The impact of decreasing U.S. hip fracture rates on future hip fracture estimates. Osteoporos Int. 2013;24:2725-8.

4. Screening for osteoporosis: U.S. Preventive services task force recommendation statement. Ann Intern Med. 2011;154:356-64.

5. Cosman F, de Beur SJ, LeBoff MS et al. Clinician's Guide to Prevention and Treatment of Osteoporosis. Osteoporos Int. 2014;25:2359-81.

6. Lim LS, Hoeksema LJ, Sherin K. Screening for osteoporosis in the adult U.S. population: ACPM position statement on preventive practice. Am J Prev Med. 2009;36:366-75.

7. Watts NB, Adler RA, Bilezikian JP et al. Osteoporosis in men: an Endocrine Society clinical practice guideline. J Clin Endocrinol Metab. 2012;97:1802-22.

8. Assessment of fracture risk and its application to screening for postmenopausal osteoporosis. Report of a WHO Study Group. No. 843. 1994 Geneva, World Health Organization. WHO Technical Report Series.

9. International Society for Clinical Densitometry. 2015 ISCD Official Positions - Adult. http://www.iscd.org/official-positions/2015-iscd-official-positions-adult/ . Updated 6-1-2015. Accessed June 9, 2017.

10. Ensrud KE, Taylor BC, Peters KW et al. Implications of expanding indications for drug treatment to prevent fracture in older men in United States: cross sectional and longitudinal analysis of prospective cohort study. BMJ. 2014;349:g4120.

11. Schousboe JT, Ensrud KE. Diagnostic criteria for osteoporosis should not be expanded. Lancet Diabetes Endocrinol. 2015;3:236-8.

12. Centre for Metabolic Bone Diseases. FRAX WHO Fracture Risk Assessment Tool. http://www.shef.ac.uk/FRAX/ . Updated 2011. University of Sheffield, UK. Accessed June 9, 2017.
13. Nayak S, Edwards DL, Saleh AA, Greenspan SL. Systematic review and meta-analysis of the performance of clinical risk assessment instruments for screening for osteoporosis or low bone density. Osteoporos Int. 2015;26: 1543-54.

14. Adler RA, Tran MT, Petkov VI. Performance of the Osteoporosis Selfassessment Screening Tool for osteoporosis in American men. Mayo Clin Proc. 2003;78:723-7.

15. Lynn HS, Woo J, Leung PC et al. An evaluation of osteoporosis screening tools for the osteoporotic fractures in men (MrOS) study. Osteoporos Int. 2008; 19: 1087-92.

16. Richards JS, Peng J, Amdur RL et al. Dual-energy X-ray absorptiometry and evaluation of the osteoporosis self-assessment tool in men with rheumatoid arthritis. J Clin Densitom. 2009;12:434-40.

17. Skedros JG, Sybrowsky CL, Stoddard GJ. The osteoporosis selfassessment screening tool: a useful tool for the orthopaedic surgeon. J Bone Joint Surg Am. 2007;89:765-72.

18. Steuart Richards J, Lazzari AA, Teves Qualler DA, Desale S, Howard R, Kerr GS. Validation of the osteoporosis self-assessment tool in US male veterans. J Clin Densitom. 2014;17:32-7.

19. Papaioannou A, Morin S, Cheung AM et al. 2010 clinical practice guidelines for the diagnosis and management of osteoporosis in Canada: summary. CMAJ. 2010;182:1864-73.

20. Blank JB, Cawthon PM, Carrion-Petersen ML et al. Overview of recruitment for the osteoporotic fractures in men study (MrOS). Contemp Clin Trials. 2005;26:557-68.

21. Orwoll E, Blank JB, Barrett-Connor E et al. Design and baseline characteristics of the osteoporotic fractures in men (MrOS) study-a large observational study of the determinants of fracture in older men. Contemp Clin Trials. 2005;26:569-85.

22. WHO Scientific Group on the assessment of osteoporosis at primary health care level. Summary meeting report, Brussels, Belgium, 5-7 May 2004. www.who.int . Updated 2007. Geneva, World Health Organization. Accessed June 9, 2017.

23. Looker AC, Melton LJ, III, Borrud LG, Shepherd JA. Lumbar spine bone mineral density in US adults: demographic patterns and relationship with femur neck skeletal status. Osteoporos Int. 2012;23:1351-60.

24. Lohman TG, Roche AF, Martorell R. Anthropometric standardization reference manual. Champaign, IL: Human Kinetics Books, 1988.

25. Pahor M, Chrischilles EA, Guralnik JM, Brown SL, Wallace RB, Carbonin P. Drug data coding and analysis in epidemiologic studies. Eur J Epidemiol. 1994; 10:405-11.

26. Hajian-Tilaki K. Receiver Operating Characteristic (ROC) Curve Analysis for Medical Diagnostic Test Evaluation. Caspian J Intern Med. 2013;4:627-35.

27. Hanley JA, McNeil BJ. The meaning and use of the area under a receiver operating characteristic (ROC) curve. Radiology. 1982;143:29-36.

28. Wright NC, Looker AC, Saag KG et al. The recent prevalence of osteoporosis and low bone mass in the United States based on bone mineral density at the femoral neck or lumbar spine. J Bone Miner Res. 2014;29:2520-6.

29. Watts NB, Leslie WD, Foldes AJ, Miller PD. 2013 International Society for Clinical Densitometry Position Development Conference: Task Force on Normative Databases. J Clin Densitom. 2013;16:472-81.

30. McGee S. Simplifying likelihood ratios. J Gen Intern Med. 2002;17:646-9

31. Pang WY, Inderjeeth CA. FRAX without bone mineral density versus osteoporosis self-assessment screening tool as predictors of osteoporosis in primary screening of individuals aged 70 and older. J Am Geriatr Soc. 2014;62:442-6.

32. Sinnott B, Kukreja S, Barengolts E. Utility of screening tools for the prediction of low bone mass in African American men. Osteoporos Int. 2006; 17:684-92

33. Schousboe JT, Taylor BC, Fink HA et al. Cost-effectiveness of bone densitometry followed by treatment of osteoporosis in older men. JAMA. 2007;298:629-37.

34. Schousboe JT, Gourlay M, Fink HA et al. Cost-effectiveness of bone densitometry among Caucasian women and men without a prior fracture according to age and body weight. Osteoporos Int. 2013;24:163-77.

35. Ito $\mathbf{K}$, Hollenberg JP, Charlson ME. Using the osteoporosis selfassessment tool for referring older men for bone densitometry: a decision analysis. J Am Geriatr Soc. 2009;57:218-24.

36. Tosteson AN, Melton LJ, III, Dawson-Hughes B et al. Cost-effective osteoporosis treatment thresholds: the United States perspective. Osteoporos Int. 2008;19:437-47.

37. Ensrud KE, Blackwell TL, Cawthon PM et al. Degree of Trauma Differs for Major Osteoporotic Fracture Events in Older Men Versus Older Women. J Bone Miner Res. 2016;31:204-7. 